\section{Diferentes estratégias de visita domiciliar e seus efeitos sobre a assistência pré-natal no extremo Sul do Brasil}

\author{
Effects of different home visit strategies on \\ prenatal care in Southern Brazil
}

\footnotetext{
1 Faculdade de Medicina, Universidade Federal do Rio Grande, Rio Grande, Brasil.

2 Programa de Pós-graduação em Epidemiologia,

Universidade Federal de

Pelotas, Pelotas, Brasil.

3 Departamento de Saúde da

Comunidade, Universidade

Federal de Santa Maria,

Santa Maria, Brasil.

${ }_{4}^{4}$ Coordenação Nacional

da Pastoral da Criança,

Curitiba, Brasil.

\section{Correspondência}

J. A. Cesar

Programa de Pós-graduação

em Epidemiologia,

Universidade Federal de

Pelotas.

Rua Marechal Deodoro 1160

3o andar, Pelotas, RS

96020-220, Brasil.

jacesar@terra.com.br
}

\section{Abstract}

This non-randomized community intervention study evaluated the impact of prenatal home visits by community health agents and volunteer leaders from the Children's Mission on prenatal care among poor pregnant women in Rio Grande, Rio Grande do Sul State, Brazil. Previously trained interviewers applied pre-coded questionnaires to the women at home, investigating demographic and reproductive characteristics, socioeconomic status, housing conditions, and prenatal care. Of the 339 pregnant women interviewed, 115 were assigned to the intervention group visited by community health agents, 116 to the group visited by volunteer leaders, and 108 to the control group. Pregnant women visited by community health agents began prenatal visits earlier than other groups, had more prenatal visits, lab tests, and clinical exams, and received more counseling on breastfeeding and iron supplementation. Participation by family members during medical consultations for pregnant women visited by volunteer leaders was higher than for community health agents. Pregnant women visited by community health agents received better prenatal care than the other groups. Home visits can improve the quality of prenatal care for poor women and increase participation by family members (mainly husbands) during the pregnancy.

Prenatal Care; Home Visit; Maternal Welfare
Juraci A. Cesar 1,2

Raul A. Mendoza-Sassi 1

Eduardo F. Ulmi 1

Marinel M. Dall'Agnol 3

Nelson A. Neumann 4

\section{Introdução}

Os níveis de mortalidade infantil no Brasil não são compatíveis com o seu potencial econômico. Países com pior renda per capita e Índice de Desenvolvimento Humano (IDH) apresentam coeficiente de mortalidade infantil bastante inferior ao do Brasil 1. Há varias razões para isto, dentre elas a oferta inadequada de assistência durante a gestação e o parto que aumenta os índices de morbimortalidade infantil, sobretudo no período neonatal 2,3,4.

Cerca de $15 \%$ das mães brasileiras não fazem uma única consulta médica durante a gestação e pelo menos um terço delas inicia o pré-natal quando não mais é possível prevenir um grande número de doenças. Estes indicadores escondem ainda importantes desigualdades regionais. $\mathrm{Na}$ Região Nordeste, por exemplo, isto ocorre para $25 \%$ das gestantes contra $7 \%$ no Sul 3 .

Estima-se que a realização de pelo menos seis consultas médicas e de exames laboratoriais e clínicos básicos, além da imunização contra tétano neonatal durante o pré-natal, propicie redução da ordem de $10 \%$ nos óbitos infantis em todo o país 3 .

Em 2002 a Pastoral da Criança criou a estratégia Laços de Amor 5. Esta estratégia consta de cartões entregues mensalmente à gestante pelo líder da Pastoral da Criança durante cada uma de suas visitas. Nestes cartões é explicado todo o desenvolvimento da criança bem como os 
cuidados que a gestante deve receber durante o pré-natal. Além disso, são mencionados todos os sinais indicativos de gravidade que requerem a busca imediata de cuidados médicos.

Em 2004, o Departamento Materno-Infantil da Universidade Federal do Rio Grande (FURG) elaborou um manual de capacitação para o agente comunitário de saúde. A partir dele, pretendia-se que os agentes comunitários de saúde informassem à gestante todos os procedimentos clínicos e laboratoriais a que ela deveria ser submetida durante o pré-natal, bem como a convencessem a realizar pelo menos seis consultas durante este período e fosse ainda imunizada contra tétano neonatal.

O presente artigo compara o efeito destas estratégias sobre o pré-natal de gestantes residentes na periferia da cidade de Rio Grande, Rio Grande do Sul, Brasil.

\section{Metodologia}

O presente estudo do tipo intervenção comunitária controlada e unicega não randomizado 6 foi realizado com mulheres de até 16 semanas de gestação residentes na periferia da cidade de Rio Grande, extremo Sul do Brasil. As entrevistas foram realizadas entre junho de 2004 e dezembro de 2005.

Três grupos de gestantes foram constituídos, sendo dois grupos-intervenção e um grupo controle. O primeiro grupo intervenção era formado por agentes comunitários de saúde, que se utilizou de material específico preparado a partir de textos recomendados pelo Ministério da Saúde. Neste material estavam descritos todos os procedimentos clínicos e laboratoriais a que a gestante deveria ser submetida mensalmente durante todo o pré-natal. Além disso, constava dos sinais de gravidade indicando a necessidade de busca imediata de cuidados médicos. Como parte deste treinamento, diversos exercícios foram elaborados e dramatizações realizadas. $\mathrm{O}$ segundo grupo intervenção foi constituído pelos líderes da Pastoral da Criança treinados na estratégia Laços de Amor 5. De acordo com a Pastoral da Criança, esta estratégia objetivava melhorar a qualidade do pré-natal oferecido às gestantes acompanhadas pelos seus líderes e aumentar a participação de familiares, sobretudo do pai da criança, na gravidez. Para tanto, em cada visita mensal, o líder entregava cartão correspondente constando todos os cuidados que ela deveria receber naquele mês por ocasião da sua consulta de pré-natal. O terceiro grupo, também formado por líderes da Pastoral da Criança, mas não capacitado na estratégia
Laços de Amor nem no material preparado para os agentes comunitários de saúde, constituiu o grupo controle. Estes três grupos foram constituídos desta forma porque praticamente todas as gestantes residentes na periferia da cidade de Rio Grande recebiam rotineiramente visita do líder da Pastoral da Criança ou do agente comunitário de saúde. Por esta razão, não foi possível randomizá-las, muito menos conseguir um grupo de gestantes que não recebesse visita domiciliar.

Para o cálculo do tamanho da amostra do presente estudo considerou-se o total de mulheres com renda familiar de até dois salários mínimos mensais que realizaram seis ou mais consultas durante o pré-natal. Com base em um estudo recentemente realizado em Rio Grande, $57 \%$ das mães nesta faixa de renda realizavam este número de consultas durante todo o prénatal 7 . Assim, se a prevalência de realização de consultas variar entre $57 \%$ e $60 \%$, se for possível aumentar em $24 \%$ a freqüência de gestantes que realizam seis ou mais consultas de pré-natal, trabalhar com um nível de significância de 95\%, poder de $80 \%$ e teste unicaudal, o estudo deveria incluir pelo menos 114 gestantes em cada grupo (Stata 9.0. Stata Corp., College Station, Estados Unidos). Estes valores já se encontram acrescidos de $10 \%$ para eventuais perdas.

Foram treinados para realizar intervenção 48 líderes da Pastoral da Criança e 48 agentes comunitários de saúde. Cada grupo de capacitação possuía 12 integrantes. Esta capacitação constou de oito horas de trabalho e de três reforços a cada 6-8 semanas, com duração de quatro horas para ambos os grupos. Durante a capacitação para a estratégia Laços de Amor, cada um dos cartões que deveria ser entregue à gestante durante a visita do líder da Pastoral da Criança era lido e seu conteúdo detalhadamente explicado. No caso do agente comunitário de saúde, o conteúdo do manual de capacitação era lido e também explicado bem como exercícios resolvidos e situações dramatizadas. Nestas situações, o agente comunitário de saúde fazia-se passar por integrantes de uma família onde havia alguma gestante.

A intervenção propriamente dita deveria ser realizada pelos líderes da Pastoral da Criança treinados na estratégia Laços de Amor e pelos agentes comunitários de saúde durante suas visitas mensais. Para as mulheres que se mostravam em dúvida quando a estarem ou não grávidas, o próprio entrevistador fazia o teste de gravidez no domicílio a partir da observação da coloração de uma fita em contato com a urina da gestante. Em caso de resultado positivo, estas gestantes eram imediatamente recrutadas para o estudo e orientadas a procurar os serviços de saúde. 
Doze entrevistadoras foram recrutadas para realizar esta coleta de dados, que consistia na aplicação de questionário quando do recrutamento da gestante e no pós-parto imediato. Este treinamento consistiu em leitura de questionários e manual de instrução, simulação de entrevistas em dupla e perante todo o grupo. Seis destes treinandos foram contratados para realizar entrevistas e os demais permaneceram como suplentes para a eventualidade de alguma substituição.

Diariamente, os entrevistadores deslocavamse para uma área previamente determinada da periferia da cidade de Rio Grande. Nesta área, visitavam todos os domicílios em busca de gestantes até o quarto mês de gravidez. Encontrando alguma, aplicava-se o questionário. Caso houvesse dúvida quanto à idade gestacional e esta pudesse ser elegível para o estudo, aplicava-se o questionário e, depois, discutia-se com o supervisor do estudo. Os questionários aplicados eram codificados pelo próprio entrevistador e diariamente entregues na sede do projeto.

Dois questionários foram aplicados a cada uma dessas gestantes, sendo o primeiro por ocasião da entrada no estudo, momento em que investigava suas características demográficas e vida reprodutiva, gravidez atual e nível sócioeconômico e condições de habitação e saneamento da sua família. O segundo questionário foi aplicado em até duas semanas após o parto e buscou investigar sobre os cuidados recebidos desde o início da gravidez até o pós-parto imediato, bem como tipo de parto e o profissional que o realizou.

Todos os questionários foram revisados e duplamente digitados através do programa Epi Info 6.04 (Centers for Disease Control and Prevention, Atlanta, Estados Unidos). Em seguida, estas digitações foram comparadas e uma delas corrigida. A análise inicial consistiu em verificar a distribuição de freqüência para cada uma das variáveis coletadas em relação ao grupo que a gestante pertencia 8 .

Realizou-se ainda análise multivariável tendo como desfecho a realização de pré-natal adequado.Considerou-se como tendo realizado pré-natal adequado a gestante que completou pelo menos seis consultas de pré-natal, dois exames de urina e glicemia, imunização contra tétano neonatal, teve a pressão arterial e ausculta de batimentos cárdio-fetal avaliadas, foi pesada e recebeu orientação sobre amamentação ao seio e suplementação com sulfato ferroso. Nesta análise utilizouse a regressão de Poisson com ajuste robusto da variância através do pacote estatístico Stata 9.0.

$\mathrm{O}$ protocolo de pesquisa foi submetido e aprovado pelo Comitê de Ética em Pesquisa em
Saúde (CEPAS) da FURG. Foi ainda assegurado a cada um dos participantes o direito de se retirar do estudo quando desejassem.

\section{Resultados}

Durante o período de recrutamento deste estudo foram identificadas 388 mulheres com suspeita de gravidez. Para 19 delas, a gravidez não se confirmou e oito sofreram aborto. Dentre as 361 restantes com gravidez confirmada, 21 não foram encontradas após o parto e uma recusouse a participar do estudo. Assim, as perdas deste estudo somaram $6,5 \%$, sendo seis pertencentes ao grupo visitado pelos agentes comunitários de saúde, quatro ao grupo visitado pelos líderes da Pastoral da Criança capacitados na estratégia Laços de Amor e 12 ao grupo dos líderes da Pastoral da Criança não capacitados, ou seja, pertenciam à Pastoral da Criança mas não haviam ainda tido qualquer contato com os Laços de Amor.

A idade das gestantes variou de 13 a 44 anos, com média de 24 anos nos três grupos estudados. A Tabela 1 mostra que havia menor proporção de gestantes adolescentes (com até 19 anos completos de idade) no grupo dos agentes comunitários de saúde e no grupo pastoral cujas líderes haviam sido capacitadas em relação ao grupo pastoral não capacitado. O percentual de gestantes da cor da pele parda e preta era maior no grupo pastoral não capacitado (34\%), contra pouco menos de $20 \%$ nos outros dois grupos, sendo esta diferença estatisticamente significativa. Quanto ao estado civil, cerca de $80 \%$ eram casadas, sendo este percentual ligeiramente menor no grupo pastoral não capacitado na estratégia. $\mathrm{O}$ número médio de moradores nos domicílios destas gestantes foi 4,1 para todos os grupos, variando de 3,9 no grupo visitado pelos agentes comunitários de saúde a 4,4 no grupo dos líderes da Pastoral da Criança não capacitados em Laços de Amor (Tabela 1).

Praticamente todas as gestantes (98\%) sabiam ler e escrever e pelo menos metade delas possuía cinco anos ou mais de escolaridade. O número médio de anos de estudo completo com aprovação variou de 7,1 anos no grupo visitado pelos agentes comunitários de saúde a 7,5 no grupo não capacitado na estratégia. Cerca de um quarto de todas elas possuía renda familiar inferior a um salário mínimo mensal (SM) e pouco mais de $60 \%$ renda familiar inferior a $2 \mathrm{SM}$. A renda familiar média nos três grupos foi de 2,2SM; cerca de metade delas, variando de $49 \%$ no grupo dos líderes da Pastoral da Criança não capacitados a $54 \%$ no grupo visitado pelos agentes comunitários de saúde, exerceu trabalho remunerado nos 12 meses que antecederam a entrevista (Tabela 1). 
Características demográficas e sócio-econômicas de gestantes residentes na periferia de Rio Grande, Rio Grande do Sul, Brasil, 2004-2005.

\begin{tabular}{|c|c|c|c|}
\hline Variável & $\begin{array}{l}\text { Agente de } \\
\text { saúde (\%) }\end{array}$ & $\begin{array}{l}\text { Líderes capa- } \\
\text { citados (\%) }\end{array}$ & $\begin{array}{c}\text { Líderes não } \\
\text { capacitados (\%) }\end{array}$ \\
\hline \multicolumn{4}{|l|}{ Idade em anos completos } \\
\hline $13-19$ & 26,1 & 25,9 & 28,7 \\
\hline $20-29$ & 53,9 & 48,3 & 50,0 \\
\hline 30 ou mais & 20,0 & 25,9 & 21,3 \\
\hline Média (desvio-padrão) & $24,3(6,2)$ & $24,7(7,1)$ & $24,4(6,9)$ \\
\hline \multicolumn{4}{|l|}{ Cor da pele } \\
\hline Branca & 82,6 & 73,3 & 65,7 \\
\hline Parda & 10,4 & 18,1 & 28,7 \\
\hline Preta & 7,0 & 0,9 & 5,6 \\
\hline \multicolumn{4}{|l|}{ Estado civil } \\
\hline Casada & 82,6 & 83,6 & 75,0 \\
\hline Solteira & 15,6 & 15,5 & 21,3 \\
\hline Outro & 1,8 & 0,9 & 3,7 \\
\hline Número de moradores & $3,9(2,0)$ & $4,0(2,2)$ & $4,4(1,8)$ \\
\hline \multicolumn{4}{|l|}{ Escolaridade da gestante em anos completos } \\
\hline $0-4$ & 18,3 & 17,2 & 15,7 \\
\hline $5-8$ & 51,3 & 50,9 & 46,3 \\
\hline 9 ou mais & 30,4 & 31,9 & 38,0 \\
\hline Média (desvio-padrão) & $7,1(3,0)$ & $7,2(6,7)$ & $7,5(2,9)$ \\
\hline \multicolumn{4}{|l|}{ Renda familiar em salários mínimos mensais } \\
\hline Menos de 1 & 23,5 & 28,4 & 23,1 \\
\hline $1-1,9$ & 33,9 & 37,1 & 36,1 \\
\hline 2 ou mais & 42,6 & 34,5 & 40,7 \\
\hline Média (desvio-padrão) & $2,2(1,8)$ & $2,1(1,9)$ & $2,2(2,0)$ \\
\hline Exerceu trabalho remunerado nos últimos 12 meses & 53,9 & 50,9 & 49,1 \\
\hline Total (n) & $100,0(115)$ & $100,0(116)$ & $100,0(108)$ \\
\hline
\end{tabular}

A Tabela 2 mostra que as gestantes visitadas pelos agentes comunitários de saúde iniciaram o pré-natal mais cedo que as demais. Esta mesma tabela mostra ainda que as gestantes acompanhadas pelos agentes comunitários de saúde realizaram um maior número de consultas de pré-natal em todos os pontos de corte utilizados: uma, seis ou nove consultas. Em média, gestantes visitadas pelos agentes comunitários de saúde realizaram cerca de duas consultas a mais em relação àquelas visitadas pelos outros dois grupos. Não houve diferença importante em relação à imunização antitetânica entre os três grupos estudados.

A Tabela 3 mostra que gestantes visitadas pelos agentes comunitários de saúde também realizaram um maior número de exames de urina, sangue, HIV e VDRL (sífilis) e tiveram a pressão arterial, ausculta cárdio-fetal e o peso mais freqüentemente avaliados que as gestantes dos demais grupos, sobretudo em relação ao grupo visitado pelo líder da Pastoral da Criança não capacitado. A realização de exame ginecológico, que incluía a coleta de material para realização da citologia (Papanicolaou), foi significativamente mais comum nos grupos visitados pelos líderes da Pastoral da Criança, capacitadas ou não nos Laços de Amor, que no grupo visitado pelos agentes comunitários de saúde. Esta mesma tabela mostra que gestantes pertencentes ao grupo dos agentes comunitários de saúde receberam com muito maior freqüência orientação sobre como amamentar ao seio, como a criança deve pegar o bico do seio, como lavar o seio e como lidar com seio ingurgitado. Finalmente, as gestantes visitadas pelos agentes comunitários de saúde foram mais comumente suplementadas com sulfato ferroso.

A participação de familiares durante as consultas de pré-natal foi maior entre gestantes vi- 
Assistência recebida entre gestantes residentes na periferia de Rio Grande, Rio Grande do Sul, Brasil, 2004-2005.

\begin{tabular}{|c|c|c|c|}
\hline Variável & $\begin{array}{l}\text { Agente de } \\
\text { saúde (\%) }\end{array}$ & $\begin{array}{c}\text { Líderes capa- } \\
\text { citados (\%) }\end{array}$ & $\begin{array}{c}\text { Líderes não } \\
\text { capacitados (\%) }\end{array}$ \\
\hline Iniciaram as consultas de pré-natal no primeiro trimestre & 60,9 & 49,1 & 43,8 \\
\hline \multicolumn{4}{|l|}{ Mês de gravidez em que iniciaram as consultas de } \\
\hline pré-natal [Média (desvio-padrão)] & $2,4(1,3)$ & $2,8(1,7)$ & $2,9(1,7)$ \\
\hline \multicolumn{4}{|l|}{ Número de consultas realizadas } \\
\hline Pelo menos 1 & 100,0 & 95,7 & 93,5 \\
\hline 6 ou mais & 86,1 & 75,9 & 69,4 \\
\hline 9 ou mais & 53,0 & 25,0 & 27,8 \\
\hline Média (desvio padrão) & $8,8(3,3)$ & $7,2(3,3)$ & $6,4(3,1)$ \\
\hline Receberam pelo menos uma dose de vacina antitetânica & 88,7 & 83,6 & 85,2 \\
\hline Total (n) & $100,0(115)$ & $100,0(116)$ & $100,0(108)$ \\
\hline
\end{tabular}

Tabela 3

Exames laboratoriais e clínicos realizados no pré-natal entre gestantes residentes na periferia de Rio Grande, Rio Grande do Sul, Brasil, 2004-2005.

\begin{tabular}{|c|c|c|c|}
\hline Variável & $\begin{array}{l}\text { Agente de } \\
\text { saúde (\%) }\end{array}$ & $\begin{array}{l}\text { Líderes capa- } \\
\text { citados (\%) }\end{array}$ & $\begin{array}{c}\text { Líderes não } \\
\text { capacitados (\%) }\end{array}$ \\
\hline \multicolumn{4}{|l|}{ Exames laboratoriais realizados } \\
\hline Urina & 99,1 & 95,7 & 92,6 \\
\hline Sangue & 99,1 & 95,7 & 93,5 \\
\hline Citopatológico colo uterino & 19,1 & 19,0 & 16,7 \\
\hline HIV & 99,1 & 95,7 & 92,6 \\
\hline VDRL (Sífilis) & 85,7 & 68,5 & 75,7 \\
\hline \multicolumn{4}{|l|}{ Exames clínicos realizados } \\
\hline Verificar pressão arterial & 100,0 & 92,2 & 88,0 \\
\hline Exame ginecológico & 32,2 & 48,3 & 54,6 \\
\hline Exame de mama & 44,3 & 36,2 & 35,2 \\
\hline Ausculta cárdio-fetal & 99,1 & 94,8 & 91,7 \\
\hline Pesagem & 99,1 & 92,2 & 90,7 \\
\hline \multicolumn{4}{|c|}{ Se durante a consulta foi ensinado como } \\
\hline Amamentar no seio & 67,0 & 50,0 & 40,7 \\
\hline Pegar o bico do seio & 66,9 & 47,4 & 41,7 \\
\hline Lavar a mama & 67,0 & 49,1 & 39,8 \\
\hline Lidar com seio ingurgitado & 62,6 & 49,1 & 42,6 \\
\hline \multicolumn{4}{|c|}{ Se durante a consulta foi recomendado uso de } \\
\hline Sulfato ferroso & 68,7 & 49,1 & 42,6 \\
\hline Vitamina & 36,5 & 31,0 & 39,8 \\
\hline Total (n) & $100,0(115)$ & $100,0(116)$ & $100,0(108)$ \\
\hline
\end{tabular}

sitadas pelos líderes da Pastoral da Criança sem Laços de Amor, ou seja, não capacitadas na estratégia (Tabela 4). Quanto à pessoa que foi junto com a gestante durante a consulta de pré-natal, não se observou diferença importante entre os grupos estudados. O mesmo ocorreu em relação ao sexo do recém-nascido, atendimento ao parto, local de nascimento, tipo de parto e peso ao nascer (Tabela 5). 
Participação de familiares nas consultas de gestantes residentes na periferia de Rio Grande, Rio Grande do Sul, Brasil, 2004-2005.

\begin{tabular}{|c|c|c|c|}
\hline Variável & $\begin{array}{l}\text { Agente de } \\
\text { saúde (\%) }\end{array}$ & $\begin{array}{c}\text { Líderes capa- } \\
\text { citados (\%) }\end{array}$ & $\begin{array}{c}\text { Líderes não } \\
\text { capacitados (\%) }\end{array}$ \\
\hline \multicolumn{4}{|c|}{ Se durante as consultas de pré-natal algum } \\
\hline familiar foi junto & 57,4 & 68,1 & 81,4 \\
\hline \multicolumn{4}{|l|}{ Quem foi junto à consulta } \\
\hline Marido & 72,7 & 68,8 & 62,5 \\
\hline Mãe & 39,4 & 40,3 & 53,0 \\
\hline Irmã/Irmão & 21,2 & 19,5 & 12,0 \\
\hline Outro familiar/Amigo & 31,8 & 39,0 & 37,3 \\
\hline Total (n) & $100,0(115)$ & $100,0(116)$ & $100,0(108)$ \\
\hline
\end{tabular}

Tabela 5

Características do recém-nascido e assistência ao parto de gestantes residentes na periferia de Rio Grande, Rio Grande do Sul Brasil, 2004-2005

\begin{tabular}{|c|c|c|c|}
\hline Variável & $\begin{array}{l}\text { Agente de } \\
\text { saúde (\%) }\end{array}$ & $\begin{array}{c}\text { Líderes capa- } \\
\text { citados (\%) }\end{array}$ & $\begin{array}{c}\text { Líderes não } \\
\text { capacitados (\%) }\end{array}$ \\
\hline \multicolumn{4}{|l|}{ Sexo } \\
\hline Feminino & 49,6 & 56,9 & 53,7 \\
\hline Masculino & 50,4 & 43,1 & 46,3 \\
\hline Nasceram no hospital & 100,0 & 98,3 & 99,1 \\
\hline \multicolumn{4}{|l|}{ Quem fez o parto } \\
\hline Médico & 84,3 & 85,3 & 87,0 \\
\hline Outros & 15,7 & 14,7 & 13,0 \\
\hline \multicolumn{4}{|l|}{ Tipo de parto que teve } \\
\hline Normal & 72,3 & 61,5 & 69,7 \\
\hline Cesariana & 27,7 & 38,5 & 29,3 \\
\hline \multicolumn{4}{|l|}{ Peso do recém-nascido (g) } \\
\hline Menos de 2.500 & 12,2 & 9,5 & 11,1 \\
\hline $2.500-3.499$ & 58,3 & 62,9 & 63,0 \\
\hline 3.500 ou mais & 29,6 & 27,6 & 25,9 \\
\hline Média (desvio-padrão) & $3.177(577)$ & $3.206(508)$ & $3.124(577)$ \\
\hline Total (n) & $100,0(115)$ & $100,0(116)$ & $100,0(108)$ \\
\hline
\end{tabular}

$\mathrm{Na}$ análise multivariável realizada somente as variáveis escolaridade e grupo a que pertencia a gestante no estudo mostraram-se fortemente associadas à realização de pré-natal adequado. Gestantes pertencentes aos grupos pastoral-intervenção e agentes comunitários de saúde apresentaram, respectivamente, razões de prevalência (RPs) de 1,07 (0,79-1,43) e 1,50 (1,16-1,94) de realizarem pré-natal adequado em relação ao grupo controle. Em relação à escolaridade observou-se que gestantes com 5-8 anos e 9 anos ou mais de estudo apresentaram, respectivamente, RPs de 1,19 (086-1,64) e 1,44 (1,04-2,01) de realização de pré-natal adequado. Estas análises foram ajustadas para diversas variáveis demográficas, sócio-econômicas, ambientais, reprodutivas e de utilização de serviços de saúde e obedeceu a modelo hierárquico 4 . 


\section{Discussão}

As gestantes visitadas pelos agentes comunitários de saúde iniciaram o pré-natal mais precocemente e realizaram um maior número de consultas e de exames clínicos e laboratoriais. As gestantes acompanhadas pelos líderes da Pastoral da Criança realizaram com maior freqüência exame ginecológico (Papanicolaou) e tiveram percentual maior de familiares participando das consultas de pré-natal em relação àquelas acompanhadas pelos agentes comunitários de saúde. Gestantes de melhor escolaridade e pertencentes aos grupos intervenção (agentes comunitários de saúde e pastoral com Laços de Amor) mostraram maior chance de realização de pré-natal adequado.

Ao interpretar estes resultados é preciso considerar algumas limitações deste estudo. Primeiro, a seleção das gestantes não foi aleatória. Isto se deve ao fato de os líderes da Pastoral da Criança e os agentes comunitários de saúde já estarem atuando nestas áreas, o que impediu a randomização. Logo, por mais semelhante que pareçam ser não se pode assegurar igualdade nas características avaliadas entre os grupos estudados. A segunda limitação diz respeito à comparação do potencial impacto de trabalhadores com disponibilidade e funções tão diferenciadas. O agente comunitário de saúde, por exemplo, é um trabalhador remunerado do serviço de saúde, atuando 40 horas por semana e oferecendo através de visitas mensais cerca de 25 cuidados em saúde a cerca de 120 famílias residentes na sua área de abrangência. O líder da Pastoral da Criança é voluntário, trabalha não mais que dois dias por mês, atende em média 12 famílias, possui um número de atribuições bem menor que o agente comunitário de saúde e lida somente com gestantes e crianças menores de seis anos. Além disso, o seu custo para o setor saúde é muito menor que o do agente comunitário de saúde. No entanto, estes trabalhadores são os únicos sem formação universitária que oferecem cuidados em saúde em nível domiciliar o que, a nosso ver, justifica esta comparação desde, claro, que consideradas estas diferenças; terceiro, as diferenças observadas poderiam ser atribuídas não somente à ação dos visitadores, mas à qualidade dos serviços oferecidos a estas gestantes, visto que o local de realização de pré-natal não foi o mesmo para todas elas. No entanto, $93 \%$ de todas elas realizaram pré-natal em postos de saúde, em geral atendidos por equipes do Programa Saúde da Família (PSF).

Há evidencia suficiente de que a oferta de cuidados em saúde em nível domiciliar melhora indicadores básicos de saúde, sobretudo em programas de pequena escala 9,10,11. Embora os agentes comunitários de saúde e os líderes avaliados pertençam a programas de abrangência nacional, as intervenções aqui mostradas ocorreram em uma área geográfica bem delimitada, portanto com todas as características de um programa de pequena escala. Ainda no caso do agente comunitário de saúde, o modelo testado foi originalmente concebido para este estudo. Logo, é de se supor que, nestas circunstâncias, sobretudo o agente comunitário de saúde, pelo elevado número de visitas que realiza e pela sua forte integração com o serviço local de saúde, seja capaz de alcançar maior impacto em suas ações 10,11.

Estudos que avaliam o impacto de visitas domiciliares sobre o pré-natal como os aqui apresentados são raros. E quando ocorrem, limitamse a comparar intervenções antes e após 12,13. Raramente encontra-se algum que, além desta comparação, utilize-se de grupo controle para avaliar o possível efeito das intervenções 10. Em geral, o impacto das vistas domiciliares ocorre sobre doenças específicas como diarréia 14, tuberculose 15 , malária 16, ou com síndromes, como as infecções respiratórias 17, e cuidados preventivos em saúde como imunização 10,13,18 e amamentação 10,13.

$\mathrm{O}$ fato de, neste estudo, as gestantes visitadas pelos agentes comunitários de saúde iniciarem o pré-natal mais precocemente e realizarem um maior número de consultas pode ser atribuído à alta freqüência de visitas domiciliares por eles realizadas, fazer parte da equipe da unidade local de saúde, ser o integrante da equipe de saúde de mais fácil acesso e por agendar consultas para todos os membros da família 10,11,19.

A realização de exames laboratoriais depende da sua disponibilidade visto que cada unidade básica de saúde dispõe de um númerolimitado de exames. Em geral, o agente comunitário de saúde conhece esta disponibilidade e avisa à gestante quando há possibilidade de realizar estes exames 19. Em relação a exames clínicos e orientações sobre tipo de parto e amamentação e dieta, por exemplo, são informações constantes na carteira da gestante. Além disso, os agentes comunitários de saúde foram orientados a incentivar as mães a conversar com o médico sobre estes assuntos quando das consultas de pré-natal. Vale lembrar também que não se avaliou se estas orientações eram adequadas ou não, mas somente se haviam sido abordadas durante consulta pré-natal. Esta capacidade de o visitador domiciliar disseminar assuntos na comunidade e posteriormente ser requisitado junto aos serviços de saúde já foi também identificada em outros estudos 11,20,21. 
É plausível também supor que os líderes tenham obtido maior sucesso no convencimento de familiares a participar mais ativamente das consultas de pré-natal visto que, na estratégia Laços de Amor 5, este tipo de abordagem é amplamente recomendado. Estas diferenças só não foram mais evidentes em função da baixa freqüência de visitas dos líderes da pastoral, visto que menos de $30 \%$ das gestantes haviam sido visitadas pelos seus respectivos líderes capacitados ou não na estratégia Laços de Amor. A baixa freqüência de visitas domiciliares por parte dos líderes parece ser um dos principais limitantes ao impacto da sua ação. Este é um aspecto que merece ser estudado em profundidade.

A melhoria dos indicadores de saúde infantil depende da extensão dos cuidados em nível domiciliar 11,22. Esta extensão passa, pelo menos no Brasil, pelos agentes comunitários de saúde e pelos líderes da Pastoral da Criança. O estudo aqui apresentado mostra claramente que estes trabalhadores têm enorme potencial para atuar na assistência à gestação. No entanto, há necessidade de capacitá-los adequadamente, oferecer suporte contínuo e melhorar sua integração com as equipes locais de saúde, fato este facilmente percebido, mas pouco referido 10,11,22. Por fim, estudos como este, apesar das limitações já mencionadas, podem contribuir na melhoria da qualidade dos cuidados por eles oferecidos e, conseqüentemente, aumentar o impacto de suas intervenções.

\section{Resumo}

Este estudo do tipo intervenção comunitária controlada unicega não randomizada teve por objetivo avaliar o impacto das visitas realizadas pelos agentes comunitários de saúde e líderes voluntários da Pastoral da Criança sobre o pré-natal de gestantes pobres em Rio Grande, Rio Grande do Sul, Brasil. Aplicaram-se questionários padronizados antes e depois do parto buscando conhecer suas características demográficas, reprodutivas, assistência recebida durante o pré-natal e nível sócio-econômico e condição de moradia. Estas gestantes foram divididas em três grupos, sendo dois grupos-intervenção e um controle. Dentre as 339 gestantes estudadas, 115 pertenciam ao grupo cuja intervenção foi realizada pelos agentes comunitários de saúde, 116 pelos líderes voluntários da pastoral e 108 pertenciam ao grupo controle. Gestantes visitadas pelos agentes comunitários iniciaram o pré-natal mais precocemente, realizaram maior número de consultas, exames clínicos e testes laboratoriais, foram mais comumente orientadas sobre amamentação e suplementadas com sulfato ferroso. A participação de familiares nas consultas de pré-natal foi maior entre gestantes visitadas pelos líderes voluntários. Visitas domiciliares podem melhorar a qualidade do pré-natal entre gestantes pobres e aumentar a participação de familiares, sobretudo do marido, na gestação.

Cuidado Pré-Natal; Visita Domiciliar; Bem-Estar Materno

\section{Colaboradores}

J. A. Cesar e R. A. Mendoza-Sassi foram responsáveis pelo delineamento, análise de dados e redação final do artigo. M. M. Dall'Agnol colaborou no levantamento bibliográfico, capacitação de agentes comunitários de saúde, elaboração de manual e auxílio na análise de dados e redação final do artigo. E. F. Ulmi realizou o controle de qualidade, comparação de digitação e auxiliou na análise preliminar e final de dados e na redação final do artigo. N. A. Neumann participou da definição do delineamento, critérios metodológicos e auxiliou na análise de dados e redação final do artigo.

\section{Agradecimentos}

Às coordenações da Pastoral da Criança e do Programa Agentes Comunitários de Saúde e Programa Saúde da Família (PACS/PSF), aos agentes comunitários de saúde, aos líderes da Pastoral da Criança, aos financiadores, às gestantes que participaram deste estudo e aos comentários da Professora Iná S. Santos por ocasião da análise dos dados e redação deste artigo. À Pastoral da Criança, Fundo das Nações Unidas para a Infância e Adolescência (UNICEF) e Programa Criança Esperança da Rede Globo de Televisão pelo financiamento. 


\section{Referências}

1. United Nations Children's Fund. The state of the world's children 2006. New York: United Nations Children's Fund; 2006.

2. Martines J, Paul VK, Bhutta ZA, Koblinsky M, Soucat A, Walker N, et al. Neonatal survival: a call for action. Lancet 2005; 365:1189-97.

3. Victora CG. Intervenções para reduzir a mortalidade infantil pré-escolar e materna no Brasil. Rev Bras Epidemiol 2001; 4:3-69.

4. Victora CG, Huttly SR, Fuchs SC, Olinto MT. The role of conceptual frameworks in epidemiological analysis: a hierarchical approach. Int J Epidemiol 1997; 26:224-7.

5. Pastoral da Criança. Guia do líder da Pastoral da Criança: da gestação aos seis anos de idade. Curitiba: Pastoral da Criança; 2007.

6. Friedman LM, Furberg CD, DeMets DL. Fundamentals of clinical trials. $3^{\text {rd }}$ Ed. New York: Springer-Verlag; 2000.

7. Cesar JA, Mendoza-Sassi R, Horta BL, Ribeiro PR, D'Avila AC, Santos FM, et al. Indicadores básicos de saúde infantil em área urbana no extremo sul do Brasil: estimando prevalências e avaliando diferenciais. J Pediatr (Rio J) 2006; 82:437-44.

8. Kirkwood BR. Essential of medical statistics. London: Blackwell Scientific Publications; 1988.

9. Berman PA, Gwatkin DR, Burger SE. Communitybased health workers: head start or false start towards health for all? Soc Sci Med 1987; 25:443-59.

10. Cesar JA, Cavaleti MA, Holthausen RS, Lima LG. Mudanças em indicadores de saúde infantil em um município com agentes comunitários: o caso de Itapirapuã Paulista, Vale do Ribeira, São Paulo, Brasil. Cad Saúde Pública 2002; 18:1647-54.

11. Haines A, Sanders D, Lehmann U, Rowe AK, Lawn JE, Jan S, et al. Achieving child survival goals: potential contribution of community health workers. Lancet 2007; 369:2121-31.

12. Cufino Svitone E, Garfield R, Vasconcelos MI, Craveiro VA. Primary health care lessons from the Northeast of Brazil: the Agentes de Saúde Program. Rev Panam Salud Pública 2000; 7:293-302.
13. Emond A, Pollock J, Costa N, Maranhão T, Macedo A. The effectiveness of community-based interventions to improve maternal and infant health in the Northeast of Brazil. Rev Panam Salud Pública 2002; 12:101-10

14. Bailey JE, Coombs DW. Effectiveness of an Indonesian model for rapid training of Guatemalan health workers in diarrhea case management. J Community Health 1996; 21:269-76.

15. Chowdhury AM, Chowdhury S, Islam MN, Islam A, Vaughan JP. Control of tuberculosis by community health workers in Bangladesh. Lancet 1997; 350:169-72.

16. Kidane G, Morrow RH. Teaching mothers to provide home treatment of malaria in Tigray, Ethiopia: a randomised trial. Lancet 2000; 356:550-5.

17. Zeitz PS, Harrison LH, López M, Cornale G. Community health worker competency in managing acute respiratory infections of childhood in Bolivia. Bull Pan Am Health Organ 1993; 27:109-19.

18. San-Sebastian M, Goicolea I, Aviles J, Narvaez M. Improving immunization coverage in rural areas of Ecuador: a cost-effectiveness analysis. Trop Doct 2001; 31:21-4.

19. Cesar JA. Community health workers in Sergipe, Brazil: implications for their future role in maternal and child health [Tese de Doutorado]. London: London School of Hygiene and Tropical Medicine; 2005.

20. Kahssay HM, Taylor ME, Berman PA. Community health workers: the way forward. Geneva: World Health Organization; 1999.

21. Opoku SA, Kyei-Faried S, Twum S, Djan JO, Browne EN, Bonney J. Community education to improve utilization of emergency obstetric services in Ghana. The Kumasi PMM Team. Int J Gynaecol Obstet 1997; 59 Suppl 2:S201-7.

22. Sundararaman T Community health-workers: scaling up programmes. Lancet 2007; 369:2058-9.

Recebido em 08/Mai/2007

Versão final reapresentada em 05/Mai/2008 Aprovado em 07/Mai/2008 